\title{
Recuit et gravure induits par laser sur silicium : expériences et simulations numériques
}

\author{
B. Bourguignon, B. Dragnea, J. Boulmer*, J.-P. Budin* et D. Débarre* \\ Laboratoire de Photophysique Moléculaire du CNRS, Bâtiment 213, Université de \\ Paris-Sud, 91405 Orsay cedex, France \\ * Institut d'Électronique Fondamentale associé au CNRS, Bâtiment 220, Université de \\ Paris-Sud, 91405 Orsay cedex, France
}

\begin{abstract}
Résumé : L'ensemble des processus se produisant pendant la fusion de surface induite par laser : diffusion de chaleur, des impuretés, ségrégation et désorption, est inclus pour la première fois dans une simulation numérique décrivant la gravure du silicium en présence de chlore. L'évolution de la surface pendant le pulse laser, les énergies de désorption depuis la surface liquide, le coefficient de ségrégation du chlore dans le silicium, sont obtenus. Les implications pour d'autres systèmes que $\mathrm{Cl} / \mathrm{Si}$ sont discutées.
\end{abstract}

\section{INTRODUCTION}

La fusion superficielle induite par laser permet de provoquer une grande variété de modifications du substrat : gravure, nettoyage, incorporation d'une impureté, et modification du profil de concentration des impuretés existantes, dans la couche ultra-mince fondue par le laser. Une amélioration des propriétés tribologiques ou des propriétés chimiques, comme la résistance à la corrosion, peut aussi résulter d'une fusion superficielle. L'effet obtenu dépend des impuretés contenues dans le (ou à la surface du) substrat.

Cette variété de modifications possibles est dûe à trois causes : l'augmentation de la probabilité de désorption entre un liquide et un solide favorise gravure et nettoyage ; l'augmentation considérable (typiquement de 7 ordres de grandeur) du coefficient de diffusion des impuretés entre phases solide et liquide favorise l'homogénéisation du profil de concentration des impuretés dans la couche fondue, et l'incorporation dans le volume (désirée ou non) de molécules adsorbées; enfin la plus ou moins grande importance du phénomène de ségrégation à l'interface liquide - solide pendant la recristallisation est le paramètre principal qui détermine le profil de concentration final (i.e. après recristallisation) des impuretés.

Du fait de la complexité et de la multiplicité des processus (diffusion de chaleur, changements de phase, diffusion d'atomes, ségrégation, désorption), l'interprétation quantitative des expériences nécessite une simulation numérique. Les processus de diffusion de la chaleur produite par l'absorption du faisceau laser, de fusion superficielle et de diffusion de dopants dans le silicium sont largement décrits dans la littérature. Par contre, la désorption n'a pas été incluse dans les simulations antérieures. Or celle-ci joue un rôle essentiel dans la gravure et le nettoyage du substrat. 
Cette étude décrit donc la première simulation numérique complète des processus intervenant pendant la fusion superficielle induite par laser, y compris la désorption. Le système choisi pour cette première simulation est le chlore sur le silicium, pour lequel des données expérimentales très complètes sont disponibles : chlore désorbé et proportion entre les différentes espèces désorbées par spectrométrie de masse à double temps de vol, chlore restant à la surface par spectroscopie Auger, chlore restant dans le volume par SIMS. Le modèle sera ultérieurement appliqué à d'autres cas, comme Ge et $\mathrm{C}$ (recuit laser de couches de $\mathrm{SiGeC}$ épitaxiées) et $\mathrm{O}$.

\section{REACTIONS DU CHLORE SUR LE SILICIUM}

Le chlore se chimisorbe sur le silicium en le passivant. En apportant de l'énergie à la surface, on observe la désorption non pas de chlore, mais de chlorure de silicium $\mathrm{SiCl}_{\mathrm{x}}$, où $\mathrm{x}$ dépend de la nature de l'énergie apportée (et de la vitesse avec laquelle on l'apporte, comme on le verra ci-dessous). Pour obtenir une gravure de la surface, il suffit d'apporter l'énergie de manière spatialement sélective (par exemple à l'aide d'un faisceau laser). Un chauffage thermique du silicium chloré conduit à la désorption de $\mathrm{SiCl}_{2}$. Au dessus de $800^{\circ} \mathrm{C}, \mathrm{SiCl}$ apparaît aussi comme produit de désorption [1]. La réaction directe de $\mathrm{Cl}_{2}$ gazeux peut aussi se produire à condition que les molécules possèdent une énergie cinétique de l'ordre de $1 \mathrm{eV}$ [2]. Cette réaction peut aussi être photoassistée, et dans ce cas elle se produit à des énergies cinétiques beaucoup plus faibles (inférieures à $0.1 \mathrm{eV}$ ). La réaction de surface conduit à la formation de $\mathrm{SiCl}_{2}[3]$.

Lorsque la surface est exposée à un gaz de chlore à une pression de $\approx 100$ mbar (correspondant à un taux de collision de 1 chaque $10 \mathrm{~ns}$ pour chaque atome de surface), l'effet d'un pulse de laser à excimère est double : il provoque à la fois l'activation de la réaction du chlore gazeux à la surface, et la désorption des chlorures de silicium provenant de l'adsorption de chlore adsorbé avant le pulse laser. Il est possible d'observer séparément ces deux types de réactivité.

La désorption photoinduite de chlorures chimisorbés est observable par exemple par spectrométrie de masse à l'aide d'un jet de chlore pulsé permettant l'adsorption de chlore avant le pulse laser. On observe expérimentalement qu'elle n'est efficace qu'au dessus du seuil de fusion superficielle du silicium ; celui-ci est mis en évidence par la très forte augmentation du coefficient de réflectivité du Si dans le rouge lorsqu'il fond (figure 1) [4]. II n'y a aucune indication que le processus ne soit pas entièrement thermique. $\mathrm{SiCl}$ et $\mathrm{SiCl}_{2}$ désorbent en proportions semblables, alors que la désorption de $\mathrm{Cl}$ et de $\mathrm{Cl}_{2}$ est négligeable [5]. L'efficacité de désorption (ou la vitesse de gravure) augmente de 4 ordres de grandeur lorsque le flux laser augmente du seuil de fusion $\mathrm{de} \approx 350 \mathrm{~mJ} / \mathrm{cm}^{2}$ à $500 \mathrm{~mJ} \mathrm{~cm}{ }^{-2}$. Elle atteint ensuite un plateau et ne varie plus avec le flux laser jusqu'au seuil d'ablation [6]. L'efficacité maximale est de 0.4 monocouche (ML) de Si par pulse, et elle ne correspond pas à l'efficacité attendue si l'on supposait que chaque atome de chlore adsorbé (1 ML) désorbe en emportant avec lui un atome de $\mathrm{Si}$ [7]. Ce résultat intriguant a conduit à mesurer la quantité de chlore restant dans le volume et à la surface après un pulse laser. Le résultat expérimental est que le chlore n'est pas mesurable en volume, mais qu'il y a environ $0.3 \mathrm{ML}$ de chlore à la surface : ceci montre qu'une fraction du chlore pénètre dans le matériau comme pour échapper à la désorption, mais qu'il y revient par ségrégation à la fin de la recristallisation [5].

La réaction photoinduite du chlore gazeux à la surface a été observée pour des flux laser inférieurs au seuil de fusion superficielle, en apportant $\mathrm{Cl}_{2}$ sur la surface par un jet 

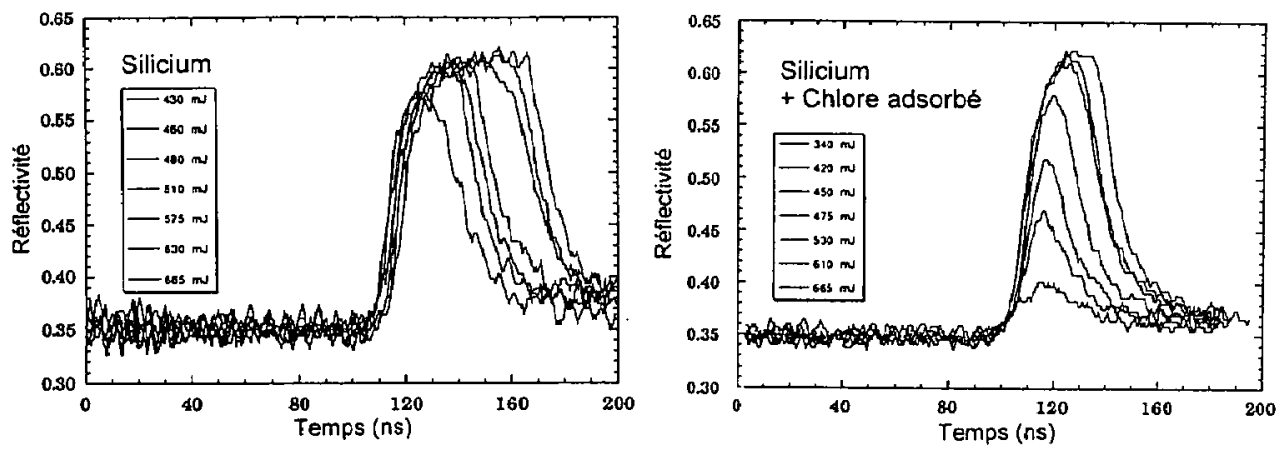

Figure 1. Evolution de la réflectivitè à $638 \mathrm{~nm}$ d'une surface de silicium pendant le pulse d'un laser à excimère en présence et en l'absence d'une monocouche de chlore adsorbé. Les transitoires augmentent en durée et en amplitude avec le flux du laser. La durée de la fusion superficielle est égale à la largeur des transitoires.

supersonique, et en détectant les produits de réaction par spectrométrie de masse [3], ou encore à pression beaucoup plus élevée, en mesurant après coup la profondeur de gravure de la surface [8]. La réaction du chlore gazeux est entièrement différente de la désorption, et elle fait intervenir des processus électroniques (mis en évidence par l'effet du niveau de dopage sur la vitesse de gravure). Elle peut se produire à des flux laser faibles (pour lesquels la désorption des chlorures chimisorbés est négligeable), et son efficacité augmente de façon constante avec le flux laser. Dans ce travail, on s'intéresse au premier processus, la désorption photoinduite de chlorures chimisorbés.

\section{CALCULS}

La simulation numérique de la fusion superficielle du silicium a fait l'objet de nombreux travaux au cours des années 1980 [9-12]. La simulation comprend la résolution numérique de l'équation de diffusion de la chaleur, en incluant le changement de phase. Il faut noter que le changement de phase est loin de se produire dans des conditions proches de l'équilibre thermique, car le gradient de température, et donc la vitesse du front de fusion ou de recristallisation, sont " grands ». " Grand » signifie que le front de changement de phase passe si vite que le matériau s'échauffe (refroidit) au dessus (au dessous) de la température de fusion avant que les atomes n'aient eu le temps d'absorber (restituer) la chaleur latente de fusion (en d'autres termes, avant que les atomes n'aient eu le temps de bouger). Il en résulte un retard à la fusion/recristallisation : la température de fusion/recristallisation est variable, l'écart avec la valeur d'équilibre atteignant $\pm 100 \mathrm{~K}$ aux flux nécessaires à la gravure. A flux laser plus élevé que dans nos expériences, il se produit des phénomènes parasites : croissance d'une phase amorphe, recristallisation explosive. Notre simulation numérique tient compte du retard à la fusion/recristallisation et elle utilise les paramètres du silicium tabulés dans la référence 12 (figure 2 ).

La désorption est traitée par une cinétique du premier et du second ordre en taux de recouvrement du chlore à la surface. La cinétique du premier ordre correspond à la désorption de $\mathrm{SiCl}$, et celle du second ordre à celle de $\mathrm{SiCl}_{2}$. Plusieurs schémas cinétiques sont envisagés. La désorption est couplée à la résolution numérique de l'équation de diffusion des atomes de chlore à l'intérieur du solide, à laquelle est également incluse la ségrégation. La 


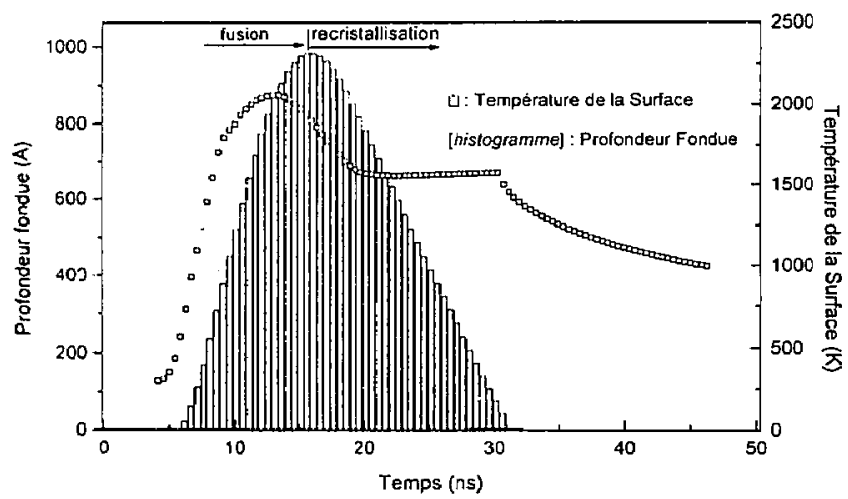

Figure 2. Evolution calculée de la température de la surface et de la profondeur fondue pendant le pulse d'un laser à excimère incident sur une surface de $\mathrm{Si}$, à $308 \mathrm{~nm}$, d'énergie $600 \mathrm{~mJ} / \mathrm{cm}^{2}$ et de durée II ns à mi-hauteur.

ségrégation à l'interface solide-liquide pendant la recristallisation est décrite dans le programme sous la forme d'un coefficient de ségrégation $\mathrm{K}_{\mathrm{s}}$. qui est égal au rapport entre les densités du chlore dans les deux phases à l'interface solide-liquide pendant la recristallision : $\mathrm{K}_{\mathrm{s}}=\mathrm{n}_{\mathrm{s}} / \mathrm{n}_{\mathrm{l}}$. La valeur de $\mathrm{K}_{\mathrm{s}}$ dépend de la vitesse de l'interface, et certains atomes comme As subissant une forte ségrégation à l'équilibre ne subissent aucune ségrégation dans les conditions du recuit laser, où l'interface se déplace typiquement à une vitesse de plusieurs mètres par seconde. Les détails de nos calculs sont donnés dans la réf. 13. Les énergies d'activation de la désorption et le coefficient de ségrégation sont les paramètres déterminées par la comparaison avec l'expérience. Il faut noter que les énergies de désorption sont connues pour la surface solide, ce qui permet de tester nos calculs.

\section{RESULTATS}

Les coefficients de diffusion des impuretés dans le silicium liquide ont une telle amplitude que la diffusion vers le volume modifie considérablement le taux de recouvrement de la surface par l'impureté. La longueur caractéristique de la diffusion varie avec le temps. En 1 ns, elle est de $40 \AA$. Au voisinage du seuil de fusion, à $350 \mathrm{~mJ} / \mathrm{cm}^{2}$, alors que la surface ne fond que pendant $3.5 \mathrm{~ns}$, le taux de recouvrement de chlore décroît ainsi des deux tiers dès que la surface fond, diminuant fortement la vitesse de désorption. A des flux plus élevés, le dépeuplement est de l'ordre de trois ordres de grandeur (figure 3). Il en résulte que la désorption ne dure effectivement qu'une fraction de nanoseconde, et que son importance dépend surtout du taux de recouvrement initial de la surface par le chlore et de la cinétique de désorption, et pratiquement pas de la ségrégation (qui ne se produit qu'après la désorption). Par contre, la quantité de chlore restant à la surface et dans le volume à la fin du pulse laser est essentiellement dépendante de la valeur du coefficient de ségrégation, et elle a une grande importance pour le rendement de désorption au pulse suivant. Le coefficient de ségrégation

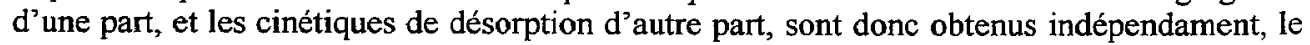
premier, à l'aide des donnée SIMS et Auger, les seconds, à l'aide des données de temps de vol et des mesures de la vitesse de gravure. 


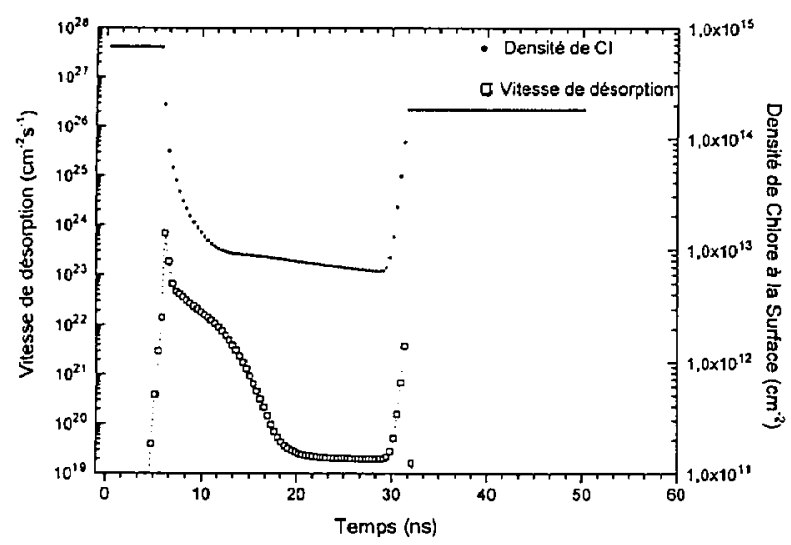

Figure 3. Dynamique de la désorption induite par fusion superficielle : évolution de la vitesse de désorption et du taux de recouvrement en chlore à la surface pendant le pulse d'un laser à excimère à $600 \mathrm{~mJ} / \mathrm{cm}^{2}$. Le dépeuplement rapide (c I ns) de la surface en chlore a pour effel de limiter la durée effective de la désorption à moins de I $n s$.

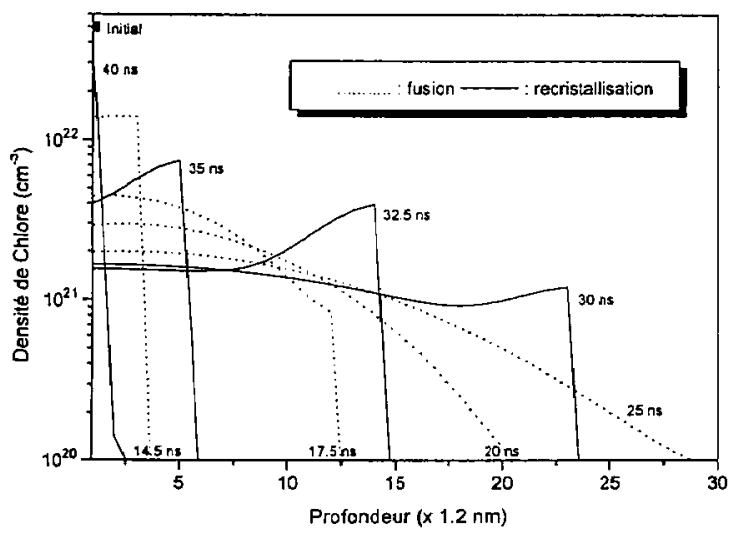

Figure 4. Evolution du profil de concentration du chlore dans le volume pendant la fusion superficielle. L'état initial est une monocouche de chlore. A noter que durant la fusion, la longueur de diffusion du chlore est d'abord plus grande que celle de la chaleur, puis plus petite (he chlore ne "remplit "plus tonte la couche fonhue après $20 \mathrm{~ns})$. Pendant la recristallisation, la ségrégation du chlore à l'interface liquide-solide se manifeste par le pic de chlore qui se déplace avec le front de recristallisation vers la sturface.

La comparaison avec l'expérience a permis d'obtenir $\mathrm{K}_{s}<0.02$ pour $\mathrm{Cl}$ à l'interface solide/liquide du silicium. Cette valeur très basse n'est qu'une limite supérieure, car Cl s'est en fait révélé indétectable dans les expériences de SIMS [5]. $\mathrm{Cl}$ est une impureté qui ne peut se placer ni en site de substitution parce que sa structure électronique ne s'y prête pas, ni en site intersticiel parce qu'il est trop gros. La simulation de la diffusion du chlore est montrée dans la figure 4 , et elle permet de représenter l'effet de « pompage $*$ d'une surface liquide sur un adsorbat, ainsi que l'《expulsion » du chlore du volume correspondant à la ségrégation. 
Il résulte de ce qui précède que si l'énergie de désorption était la même sur la surface liquide et sur la surface solide, la fusion superficielle aurait pour effet de diminuer la désorption des espèces chlorées : l'inverse est observé expérimentalement, et c'est parce que l'énergie de désorption décroît sur la surface liquide par rapport à la surface solide. La simulation des résultats expérimentaux pour différents schémas cinétiques de désorption de $\mathrm{SiCl}$ et $\mathrm{SiCl}_{2}$ conduit à des énergies de désorption plus basses de $0.5 \pm 0.2 \mathrm{eV}$ sur la surface liquide par rapport à la surface solide. Cet abaissement correspond au fait que sur une surface liquide, l'environnement de la molécule qui désorbe s'adapte rapidement pour minimiser l'énergie potentielle, tandis que sur une surface solide la désorption laisse un site réactif très énergétique. Cette valeur est aussi à rapprocher de la chaleur latente de fusion $\mathrm{du} \mathrm{Si}$, qui est égale à $0.52 \mathrm{eV}$ par atome de $\mathrm{Si}$. Les énergies de désorption obtenues pour $\mathrm{SiCl}$ sont de 2.7 $\mathrm{eV}$ sur le solide (expérimentalement : $2.45 \mathrm{eV}$ ) et de $1.95 \pm 0.15 \mathrm{eV}$ sur le liquide. Celles de $\mathrm{SiCl}_{2}$ sont de $3.7 \pm 0.1 \mathrm{eV}$ sur le solide (expérimentalement : $3.61 \mathrm{eV}$ ) et $3.15 \pm 0.15 \mathrm{eV}$ sur le liquide.

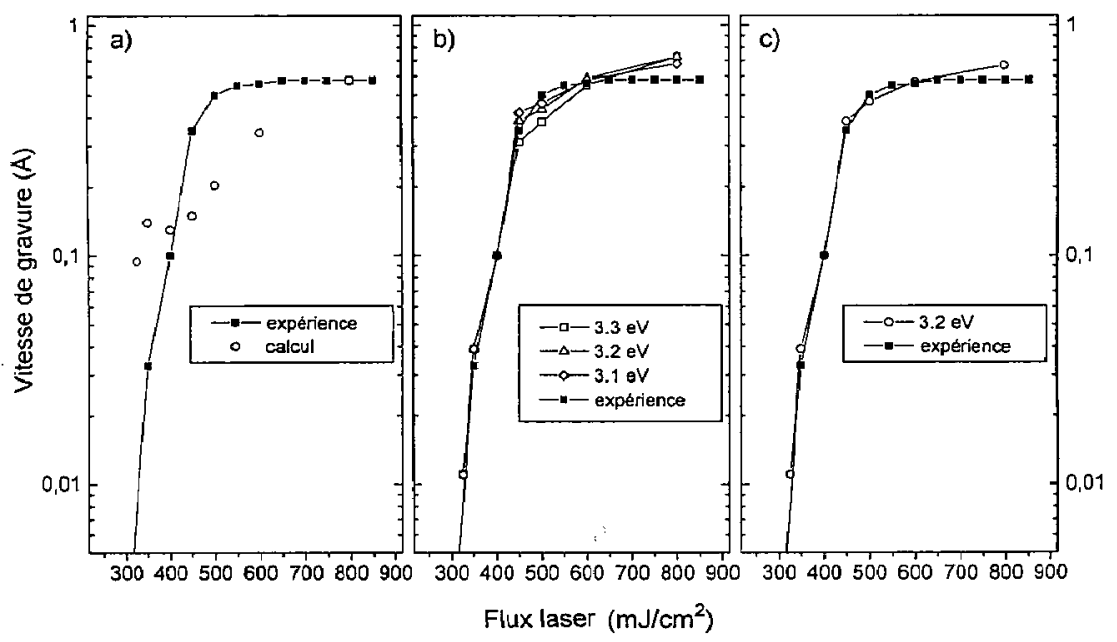

Figure 5. Efficacité de désorption (exprimée ici comme la vitesse de gravure ( $\AA$ /pulse)), mesurée et calculée pour diverses cinétiques de désorption, en fonction de l'énergie du laser : a) $2.15 \mathrm{eV}$ pour le solide et le liquide (ler ordre seulement) ; b) $3.7 \mathrm{eV}$ pour le solide (second ordre seulement) et $2.0 \mathrm{eV}$ pour le premier ordre et comme indiqué sur la figure et le second ordre sur la surface liquide ; c) $3.7 \mathrm{eV}$ pour le solide (second ordre seulement) et 2.0 et $3.2 \mathrm{eV}$ pour le premier et le second ordre sur la surface liquide. Premier et second ordres sont couplés diffétemment dans b) et c) (voir réf. [13]). La cinétique dh Ier ordre seule ne permet pas de reproduire la saturation observée expérimentalement au dessus de $500 \mathrm{~mJ} / \mathrm{cm}^{2}$.

D'autres détails de la cinétique de désorption ont été obtenus. La désorption du premier ordre ne permet pas de rendre compte à elle seule des données expérimentales (figure 5). La saturation observée de la vitesse de gravure avec le flux laser ne peut pas être expliquée sans faire intervenir la désorption de second ordre. En effet, augmenter le flux laser augmente la température maximale, la vitesse de désorption et la durée de la fusion, mais ne modifie pas beaucoup la diffusion vers le solide. Au contraire, la contribution des temps longs à la désorption de $\mathrm{SiCl}$ (queue de la vitesse de désorption sur la figure 3) augmente avec le flux laser par rétrodiffusion partielle de $\mathrm{Cl}$ vers la surface qui est trop dépeuplée en chlore par rapport au volume. L'introduction d'une composante de second ordre, correspondant à $\mathrm{SiCl}_{2}$, 
diminue cet effet car $\mathrm{SiCl}_{2}$ a une queue de désorption négligeable, la densité de chlore étant vite insuffisante pour permettre la désorption de $\mathrm{SiCl}_{2}$. Le rapport de branchement (expérimentalement égal à $1.3 \pm 0.3$ [5]) entre $\mathrm{SiCl}$ (forte énergie d'activation) et $\mathrm{SiCl}_{2}$ (plus faible énergie d'activation) s'explique ainsi par le fait que la désorption de $\mathrm{SiCl}_{2}$ est paradoxalement limitée par la diffusion sur la surface liquide. La raison physique de la saturation de la vitesse de gravure avec le flux est qu'il suffit en première approximation de fondre la surface pendant 1 ns pour induire la désorption de la fraction de chlore qui "préfère » désorber plutôt qu'entrer dans le volume: Ies millijoules supplémentaires ne servent à rien du point de vue de l'efficacité de la gravure.

La contamination du silicium par la diffusion du chlore vers le volume est limitée par la ségrégation. La contamination résiduelle peut être diminuée par d'autres pulses laser. La fraction de chlore ramenée à la surface par un pulse sera désorbée avec la même efficacité que la monocouche initiale, mais le chlore resté dans le volume sera plus difficile à désorber puisqu'il a moins de chances de diffuser vers la surface. Le calcul montre que pour le chlore la fraction désorbée décroît de $2 / 3$ pour une monocouche à $1 / 3$ après 3 pulses, et qu'elle reste stable ensuite, la forme de la distribution de chlore en profondeur se stabilisant en 3 pulses (figure 6). Pour d'autres atomes, l'efficacité du nettoyage laser dépendra essentiellement à la fois de l'énergie de désorption (c'est évident), mais aussi de manière essentielle du coefficient de ségrégation, car c'est lui qui détermine le rapport en fin de pulse entre la fraction adsorbée (facile à désorber) et la fraction «dissoute " dans le volume (difficile à désorber). Le carbone a un comportement semblable au chlore, mais pour un atome ne "ségrégeant" pas, comme $O$, il en va tout autrement, car seulement une faible fraction des atomes peut se trouver au voisinage de la surface. Il faut moins de dix pulses (soit une fraction de seconde) pour nettoyer par laser le silicium de son carbone, et quelques milliers pour le nettoyer de son oxygène après une exposition à l'air.

\section{CONCLUSIONS}

Ce travail est la premier où on inclut la désorption à une description quantitative de la fusion de surface induite par laser. La simulation est appliquée à la gravure du silicium par le chlore, comme premier système d'application, ce système étant très bien connu et étant dominé par la désorption. L'ensemble des données disponibles (Auger, SIMS, spectrométrie de masse par double temps de vol) contraint suffisamment la simulation pour que les résultats soient sans ambiguité. En particulier, le coefficient de ségrégation et les énergies de désorption sont déterminés indépendamment. La diffusion vers le volume est confirmée comme étant l'un des principaux facteurs limitant la vitesse de gravure, l'autre étant la stoecchiométrie, $\mathrm{SiCl}$ et $\mathrm{SiCl}_{2}$ désorbant en proportions semblables. La ségrégation du chlore à l'interface liquide-solide du silicium reste très forte, même à des vitesses de recristallisation de $6 \mathrm{~m} / \mathrm{s}$. Des détails concernant la dynamique de la surface pendant le pulse laser sont aussi obtenus. Ainsi, la désorption ne se produit efficacement que pendant les tout premiers instants $(<1 \mathrm{~ns})$ de la fusion superficielle.

Dans le domaine de la microélectronique, d'autres atomes que $\mathrm{Cl}$ ont une grande importance, comme par exemple B, As, $\mathrm{Sb}, \ldots$ comme dopants, Ge et $\mathrm{C}$ dans les couches épitaxiées de $\mathrm{SiGeC}\left(\mathrm{Si}_{1-x-y} \mathrm{Ge}_{x} \mathrm{C}_{y}\right)$, O comme constituant principal de la silice avec Si luimême, ou $C$ et $O$ en tant qu'impuretés du silicium. Tous ces atomes peuvent être «manipulés " par laser [14]. Il ressort de nos simulations que les paramètres essentiels sont le coefficient de ségrégation et l'énergie de désorption. Celle-ci dépend de manière critique de la nature de la molécule susceptible de désorber. L'extension de ce travail passe par de 


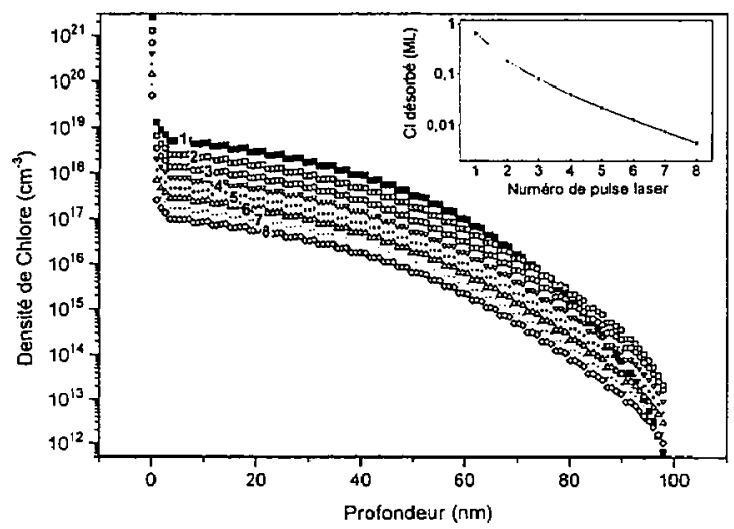

Figure 6. Nettoyage laser : profil de concentration du chlore à la fin de chaque pulse laser dans une série de huit pulses, et (en insert) efficacité de désorption en monocouche (ML) de chlore en fonction du numéro du pulse. L'état initial est une monocouche de chiore.

nouvelles expériences sur le silicium en ultra-vide. En effet, pour être exacte, la simulation numérique demande que les coefficients optiques (absorption) et thermiques (capacite calorifique et conductivité thermique) du silicium soient suffisamment bien connus. Ce n'est pas tout à fait le cas, comme le montre notre mesure des réflectivités transitoires du silicium et du silicium chloré (figure 1), qui sont significativement différentes : une monocouche d'adsorbat modifie les propriétés optiques et/ou thermiques. Or, les coefficients utilisés dans la littérature ont été déterminés dans des expériences laser-surface réalisées à l'air [11]. Un traitement complet des phénomènes décrits ici nécessitera la mesure des paramètres optiques et thermiques du silicium sous ultra-vide, la modélisation de l'influence des adsorbats sur ces propriétés, et le traitement couplé de la diffusion des atomes et de la chaleur. Ce travail est en cours dans notre équipe. L'application de notre simulation à des cas tels que $\mathrm{SiGeC}$ sera ensuite réalisée.

\section{Références}

[1] J. Matsuo, F. Yannick, K. Karahashi, Surf. Sci. 283 (1993) 52-57.

[2] F. X. Campos, G. C. Weaver, C. J. Waltman and S. R. Leone, J. Vac. Scr. Technol. B 10 (1992) $2217-2222$.

[3] Q-Z. Qin, Y.-L. Li, P.-H. Lu, Z. Zhang, Z. Jin and Q.-K. Zheng, J. Vac. Sci. Technol. B 10 (1992) $201-205$.

[4] J. Boulmer, B. Bourguignon, J. Budin, D. Débarre, A. Desmur, J. Vac. Sci. Techrol. A 9 (1991) 2923-2927.

[5] B. Bourguignon, M. Stoica, B. Dragnea, S. Carrez, J. Boulmer, J.-P. Budin, D. Débarre, A. Aliouchouche, Sirf. Sci. 338 (1995) 94-110

[6] J. Boulmer, B. Bourguignon, J.-P. Budin and D. Débarre, Appl. Surf. Sci., 43 (1989) 424-431.

[7] A. Aliouchouche, J. Boulmer, B. Bourguignon, J.-P. Budin, D. Débarre and A. Desmur, Appl. Surf. Sci., 69 (1993) 52-58.

[8] D. Débarre, A. Aliouchouche, J. Boulmer, B. Bourguignon, J.-P. Budin and S. Carrez, Am. Phys., 19 (1994) CI-245-CI-252.

[9] R.F. Wood, Phys. Rev. B, 25 (1982) 2786-2797.

[10] S. U. Campisano, Appl. Phys. A, 30 (1983) 195-206

[11] G. E. Jellison, Ir., A. Modine, Phys. Rev. B, 27 (1983) 7466-7471

[12] S. De Unamuno, E. Fogarassy, Appl. Surf. Sci., 36 (1989) 1-7.

[13] B. Dragnea, J. Boulmer, J.-P. Budin, D. Débarre and B. Bourguignon, Phys. Rev. B, accepté.

[14] A. Desmur, B. Bourguignon, J. Boulmer, J.-B. Ozenne, J.-P. Budin, D. Débarre, A. AJiouchouche, J. Appl. Phys., 76 (1994) 308 I-3087. 